\title{
Nanocompósitos de PEAD/PEBDL - Avaliação da Esfoliação da Argila Organofílica Pela Aplicação do Modelo de Nielsen e das Propriedades Mecânicas, Ópticas e Permeabilidade
}

\author{
Ana R. Morales, Carolina V. M. da Cruz, Leila Peres \\ Departamento de Tecnologia de Polímeros, UNICAMP \\ Edson N. Ito \\ Departamento de Engenharia de Materiais, UFRN
}

\begin{abstract}
Resumo: Uma blenda de polietileno de alta densidade e polietileno de baixa densidade (PEAD/PEBDL) comumente utilizada em filmes de embalagem foi modificada com argila montmorilonita organofílica comercial para obter nanocompósitos com melhores propriedades de barreira. Amostras com 5 e 7,5\% de argila (em massa) foram estudadas em diferentes condições de processamento. Filmes produzidos com os nanocompósitos foram avaliados quanto à dispersão da argila por difração de raio X e Microscopia Eletrônica de Transmissão. As propriedades de barreira dos filmes foram determinadas pelas medições de permeabilidade ao oxigênio e ao vapor d'água. As propriedades mecânicas e ópticas também foram determinadas. Observou-se uma estrutura com a argila parcialmente esfoliada e intercalada. A permeabilidade ao oxigênio diminuiu significativamente enquanto a permeabilidade à água foi pouco influenciada. As propriedades mecânicas e a transparência dos filmes foram pouco alteradas. O modelo de Nielsen foi aplicado para os dados de permeabilidade relativa e demonstrou ser um ótimo recurso para a avaliação do grau de esfoliação da argila para as diferentes amostras, que foram confirmadas pelas análises realizadas em microscopia eletrônica de transmissão.
\end{abstract}

Palavras-chave: Nanocompósitos, blendas poliméricas, propriedades de barreira, montmorilonita.

\section{PEAD/PEBDL Composites - Evaluation of the Exfoliation of Organophilic Clay Using the Nielsen Model and of the Mechanical, Optical and Permeability Properties}

\begin{abstract}
A typical high density polyethylene and linear low density polyethylene (HDPE/LLDPE) blend used in flexible packaging was modified with commercial organophylic montmorilonite clay to obtain nanocomposites with superior barrier properties. Samples with 5 and $7.5 \%$ of clay were prepared under different processing conditions. Films produced from the nanocomposites were evaluated in terms of oxygen and water vapor permeation and also by X-ray diffraction and Transmission Electron Microscopy. Tensile and optical properties were also evaluated. The clay showed to be intercalated and partially exfoliated. The oxygen permeation was mainly reduced and the vapor permeation was weakly modified. The mechanical properties and transparency did not change significantly. Nielsen's model was applied considering the relative permeability coefficient. This method was able to give an estimate of the exfoliation state of the samples, supported by the transmission electron microscopy.
\end{abstract}

Keywords: Nanocomposites, montmorillonite, barrier properties, polymeric blends.

\section{Introdução}

A utilização de argilas organofílicas em polímeros para a obtenção de nanocompósitos com propriedades especiais tem sido amplamente apresentada na literatura recente ${ }^{[1-3]}$.

Uma grande aplicação de interesse comercial está na obtenção de filmes com melhores propriedades de barreira, já que a redução da permeabilidade causada pela presença de camadas esfoliadas de argilas leva à possibilidade de novas aplicações ou mesmo à redução de espessura objetivando redução de custo econômico e ambiental das embalagens.
Embalagens flexíveis são amplamente empregadas na indústria de alimentos. Componentes sempre presentes nas estruturas simples são as poliolefinas e suas blendas. Uma blenda utilizada em filmes monoorientados de uso comercial é a de PEAD/PEBDL (polietileno de alta densidade e polietileno de baixa densidade linear). Os polietilenos são, por natureza, apolares, e excelentes barreira para a umidade e vapor de água, mas são pobres quando se trata de barreira ao oxigênio. Neste trabalho uma blenda de PEAD/PEBDL foi utilizada para a obtenção de nanocompósitos a partir

Autor para correspondência: Ana R. Morales, Departamento de Tecnologia de Polímeros, Faculdade de Engenharia Química, UNICAMP, 
da incorporação de montmorilonita modificada com sal quaternário de amônio.

Neste novo conceito de modificação de polímeros, aspectos associados à quantidade de argila, utilização de compatibilizantes e condições de processamento são a chave para a boa esfoliação da argila. Esta esfoliação é necessária para o desempenho esperado e para viabilidade do material. Técnicas como difração de raio X e MET (Microscopia Eletrônica de Transmissão) são utilizadas pra avaliar se a argila foi devidamente dispersa e se um bom grau de esfoliação foi conseguido.

A alteração de uma propriedade de desempenho de um nanocompósito relativo à sua matriz base é um forte indicativo das condições morfológicas da argila. Isto, somado às técnicas convencionais podem fornecer importantes dados para a evolução de tais sistemas.

Considerando-se as propriedades de barreira, entende-se que as camadas impermeáveis da argila forçam o permeante a percorrer um caminho tortuoso através do nanocompósito. Tem sido relatado na literatura que a permeabilidade de gases pode ser reduzida de 50 a 500 vezes mesmo para baixos teores de nanoargilas ${ }^{[4]}$. Os principais estudos foram feitos com oxigênio, gás carbônico e nitrogênio para aplicações em embalagens de alimentos e de bebidas carbonatadas.

O processo de permeação de gases através de uma membrana orgânica, como um filme polimérico, é um processo complexo que consiste de 3 processos: a sorção das moléculas do gás na superfície da membrana; a dissolução de gás dentro da membrana, a difusão, e finalmente a desorção do gás da superfície oposta do filme ${ }^{[5]}$.

A propriedade de permeabilidade é medida diretamente por células experimentais, onde a membrana com uma dada espessura e área divide a célula em dois compartimentos. No primeiro compartimento começa a circular o gás permeante a uma dada pressão. Com o tempo o gás começa a permear pela membrana e a concentração do gás aumenta, no outro compartimento, até que se estabelece um fluxo constante, em estado estacionário, quando se faz a determinação da permeabilidade.

Em polímeros semicristalinos, o tamanho e a forma dos cristalitos, a estrutura cristalina e o grau de cristalinidade têm uma grande importância no processo de permeação. Assume-se basicamente que os cristalitos consistem na fase impermeável para a molécula gasosa e que a difusão ocorre somente na fase amorfa.

Nos nanocompósitos o mecanismo de transporte dos gases é muito similar à de um polímero semicristalino. $\mathrm{O}$ nanocompósito de argila é considerado ser constituído por uma fase permeável (matriz polimérica) na qual está contida uma fração de nanoplaquetas impermeáveis dispersas. Existem aí três fatores principais que influenciam a permeabilidade de um nanocompósito: a fração volumétrica das nanoplaquetas; a orientação relativa à direção da difusão e a razão de aspecto das plaquetas.

O mecanismo de transporte dentro da matriz polimérica segue a lei de Fick e, portanto a matriz mantém as mesmas propriedades do polímero puro. Portanto é esperada uma redução na solubilidade no nanocompósito pela redução no volume da matriz polimérica assim como uma diminuição na difusão devido a um caminho muito mais tortuoso para as moléculas que estão neste processo de difusão. Como tipicamente a fração volumétrica da argila é baixa, o fator de redução de difusão pela tortuosidade assume papel principal, e este está intimamente conectado com a forma das nanoplaquetas e com seu grau de dispersão, ou seja, o grau de delaminação da argila.

Os termos intercalação e esfoliação são frequentemente utilizados para descrever os estados de morfológicos de delaminação das argilas.

A Figura 1 mostra, esquematicamente, os diferentes graus de delaminação (ou esfoliação), respectivos aos valores da espessura das nanoplaquetas $\mathrm{W}$.

Um modelo simples para descrever a permeabilidade para um arranjo regular de placas dispersas em uma membrana foi descrito por Nielsen e está apresentado na Figura $2^{[6]}$.

$\mathrm{Na}$ Figura 2, as nanoplaquetas são uniformemente dispersas e consideradas como sendo retangulares com largura, L, e espessura, W e a sua orientação é perpendicular ao sentido da difusão.

Nielsen estipulou que o coeficiente de solubilidade, S, dos nanocompósitos como Equação 1:

$$
\mathrm{S}=\mathrm{S}_{0}(1-\phi)
$$

onde $\mathrm{S}_{0}$ é o coeficiente de solubilidade do polímero puro, e $\phi$ é a fração volumétrica das nanoplaquetas que estão dispersas

Aumento de agregação (intercalação)
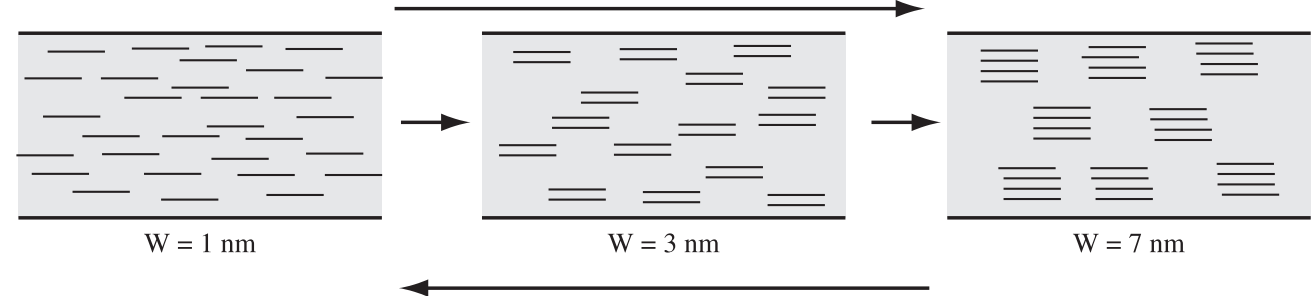

$\mathrm{W}=7 \mathrm{~nm}$

Aumento de delaminação (esfoliação)

Figura 1. Graus de esfoliação e espessura W das nanoplaquetas dispersas ${ }^{[6]}$. 


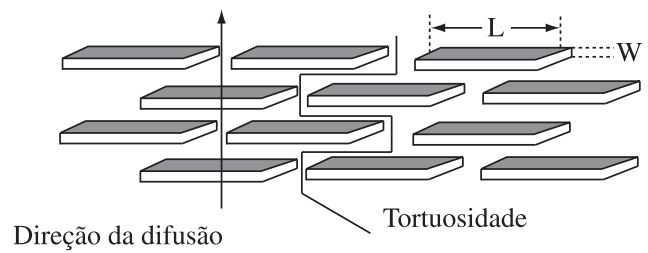

Figura 2. Modelo de permeabilidade proposto por Nielsen ${ }^{[6]}$.

na matriz (nanocarga). Neste modelo a solubilidade é independente das características morfológicas das fases.

As nanoplaquetas agem como barreiras impermeáveis para a difusão das moléculas, forçando as moléculas a seguir por caminhos mais tortuosos, consumindo mais tempo. Portanto, o coeficiente de difusão D é influenciado pela tortuosidade, $\tau$ Equação 2:

$$
\mathrm{D}=\mathrm{D}_{0} / \tau
$$

onde $\mathrm{D}_{0}$, é o coeficiente de difusão do polímero puro, e o fator $\tau$ depende da razão de aspecto, da forma e da orientação das nanoplaquetas, estando definido como Equação 3:

$$
\tau=l^{\prime} / 1
$$

onde l' é a distância que molécula deve percorrer para difundir-se através da membrana quando as nanoplaquetas estão presentes e lé a espessura da membrana.

$\mathrm{O}$ coeficiente de permeabilidade de uma membrana, $\mathrm{P}$, é produto do coeficiente de difusão $\mathrm{D}$ pelo coeficiente de solubilidade S Equação 4:

$$
\mathrm{P}=\mathrm{D} . \mathrm{S}
$$

Rearranjando as Equações 1, 2 e 4, obtém-se Equação 5:

$$
\frac{\text { P nanocompósito }}{\mathrm{P} \text { matriz }}=\frac{1-\phi}{\tau}
$$

O comprimento prolongado da difusão l' é estimado considerando que cada nanoplaqueta contribui como um aumento na média de $\mathrm{L} / 2$ na distância de difusão. $\mathrm{Se}<\mathrm{N}>$ é o número médio de nanoplaquetas que o soluto encontra então a distância de difusão através da membrana será Equação 6:

$$
l^{\prime}=1+<\mathrm{N}>\mathrm{L} / 2
$$

Desde que $<\mathrm{N}>=1 . \phi / \mathrm{W}$, assim o fator tortuosidade $\tau$ se torna Equação 7:

$$
\tau=1+\frac{\mathrm{L} . \phi}{2 \mathrm{~W}}
$$

Então, rearranjando Equação 5 (Equação 8):

$$
\frac{\mathrm{P} \text { nanocompósito }}{\mathrm{P} \text { matriz }}=\frac{1-\phi}{1+\frac{\alpha \phi}{2}}
$$

onde $\alpha=\mathrm{L} / \mathrm{W}$ é a razão de aspecto das nanoplaquetas de argila.
Direção do fluxo do gás permeante
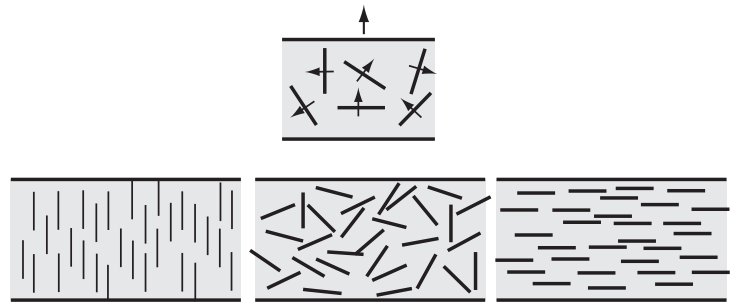

Figura 3. Representação da orientação das nanoplaquetas de argila na membrana de permeação ${ }^{[4]}$.

A Equação 8 prediz que a permeabilidade dos nanocompósitos diminui com o aumento de $\phi$ e $\alpha$.

Uma modificação da Equação (8) envolve a introdução do fator de orientação $S$, que corrige a razão de aspecto ortogonal (em relação à direção do fluxo do gás permeante) considerando a orientação média através da membrana ${ }^{[6]}$ Equação 9:

$$
\mathrm{S}=<3 \cos ^{2} \theta-1>/ 2
$$

onde $\theta$ é o ângulo entre a orientação média das nanoplaquetas e a direção do fluxo do gás permeante. Portanto para uma orientação perfeitamente paralela à superfície da membrana $\theta=0$ e $S=1$; se a orientação for perfeitamente perpendicular à superfície da membrana, $\theta=\pi / 2$ e $S=-1 / 2$ e $\theta=\pi / 4$ e $S=1 / 4$ para a orientação randômica. Desta forma a Equação (8) é modificada para Equação 10:

$$
\frac{\text { P nanocompósito }}{\mathrm{P} \text { matriz }}=\frac{1-\phi}{1+\phi \alpha(\mathrm{S}+1 / 2) / 3}
$$

Uma representação gráfica das possíveis orientações das nanoplaquetas de argila é apresentada na Figura 3.

Para nanoplaquetas planas perfeitamente orientadas perpendicularmente ao fluxo do gás permeante a Equação (10) reverte-se na Equação (8). Outras modificações do modelo de Nielsen são apresentadas na literatura considerandose parâmetros como ângulo de disposição das plaquetas de argila, forma geométrica das plaquetas de argila, mas segundo Choudalakis $^{[7]}$, o modelo de Nielsen simplificado tem se mostradoextremamenteeficientenaprevisãodapermeabilidade de nanocompósitos de argilas que têm alta razão de aspecto. Neste trabalho, assumiu-se o modelo simplificado de Nielsen, com a orientação das nanoplaquetas paralelas à superfície da membrana, considerando-se a condição mais provável obtida durante o processamento do filme por extrusão. A partir desta consideração utilizou-se a Equação (8) para os resultados do coeficiente de permeabilidade a fim de avaliar o grau de delaminação das argilas, ou seja, os valores de $\alpha$.

\section{Experimental}

\section{Materiais}

A blenda polimérica estudada foi composta de $80 \%$ de PEAD AC-59 (MFI: 0,75 g/10 minutos, $\mathrm{d}: 0,960 \mathrm{~g} / \mathrm{cm}^{3}$ ) da 
Braskem e 20\% de PEBDL LH-0828 (MFI: 0,80 g/10 minutos, d: $\left.0,920 \mathrm{~g} / \mathrm{cm}^{3}\right)$ da Braskem. A argila utilizada foi uma montmorilonita comercial modificada com sal quaternário de amônio denominada Nanomer 1.44P da Nanocor. O agente de compatibilização interfacial utilizado foi um polietileno de baixa densidade linear enxertado com anidrido maleico (PEBDL-g-AM), Fusabond E MX 110D (MFI:23 g/10 minutos, d: 0,930 g/cm ${ }^{3}$ e Tm: $122{ }^{\circ} \mathrm{C}$ ) da DuPont.

\section{Preparação dos nanocompósitos}

Concentrados de argila (masterbatches) com o agente compatibilizante foram preparados em extrusora dupla rosca co-rotante com o diâmetro 25 mm, modelo ZSK 25 Mega Compounder, L/D de 36, da marca Werner Pfleiderer. As composições e condições de processamento estão descritas na Tabela 1.

Os masterbatches foram diluídos nas concentrações em massa de 5 e 7,5\% de argila em PEAD/PEBDL (80/20) diretamente na extrusora balão para confecção de filmes, dando origem, respectivamente, aos filmes denominados nano1 e nano2; nano3 e nano4; e nano5 e nano6, com espessura aproximada de $40 \mu \mathrm{m}$.

\section{Caracterização das propriedades}

Difração de raio X: Foi utilizado um Difratômetro de raio $X$ Shimadzu modelo XRD 7000 , varredura de 1,4 a $50^{\circ}$

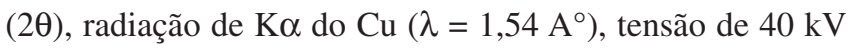
e corrente de $30 \mathrm{~mA}$, para avaliação dos picos referentes ao plano 001 da argila para identificar a ocorrência de aumento do espaçamento basal e avaliação do estado de delaminação da argila (intercalação ou esfoliação).

Permeabilidade ao oxigênio: Filmes com área de $50 \mathrm{~cm}^{2}$ e condicionados por 8 horas em dessecador com sílica, foram analisados segundo normas ASTM D3985-02 e ASTM F1927-98e1, em equipamento Oxtran 2/20, para obtenção da taxa de permeabilidade ao oxigênio (TPO).

Permeabilidade ao vapor de água: Filmes com área de $50 \mathrm{~cm}^{2}$ e condicionados por 8 horas em dessecador com sílica foram analisados segundo norma ASTM F 1249-01, em equipamento Permatran 3/33, para obtenção da taxa de permeabilidade ao vapor d'água (TPVA).

Microscopia Eletrônica de Transmissão (TEM): As amostras dos nanocompósitos nano 5 e nano 6 foram observadas por TEM para verificação da morfologia da amostras estudadas. As amostras foram previamente cortadas criogenicamente com ultramicrômetro RMC modelo MT-7000 e analisadas em equipamento Philips CM120, operando a uma voltagem de $120 \mathrm{kV}$.

Análise termogravimétrica (TGA): Foi utilizado equipamento da Mettler TGA 851, com taxa de aquecimento de $10{ }^{\circ} \mathrm{C} / \mathrm{min}$, atmosfera inerte de $\mathrm{N}_{2}, 50 \mathrm{~mL} / \mathrm{min}$, para determinação da quantidade de fase inorgânica na argila, dado necessário para o cálculo da fração volumétrica da fase impermeável no sistema.

Propriedades mecânicas: As propriedades de módulo de elasticidade e de resistência à tração máxima foram determinadas segundo ASTM D882-02, em máquina universal de ensaios Emic DL 2000 a 500 mm/min.

Propriedades óticas: Para verificar a transparência, os filmes foram analisados quanto à transmissão de luz em espectrofotômetro de UV- Visível, Varian, modelo Cary 1G.

\section{Resultados e Discussão}

Difração de raio X: Os difratogramas obtidos são apresentados na Figura 4. No estudo da intercalação da argila os picos de interesse aparecem na região de baixo ângulo. Os picos característicos da argila presente no trabalho encontram-se entre $3^{\circ}$ e $4^{\circ}$, e entre $7^{\circ}$ e $8^{\circ}$.

A argila montmorilonita Nanomer $1.44 \mathrm{P}$ apresentou dois picos evidentes de difração, $3,88^{\circ}$ e $7,12^{\circ}$, os quais correspondem aos espaçamentos basais de 2,28 e 1,24 nm, respectivamente. $\mathrm{O}$ pico presente a $3,88^{\circ}$ corresponde ao plano 001 da argila e está associado às galerias que são expandidas pela presença do sal orgânico e o pico a 7,12 pode ser considerado relativo plano 002 da Nanomer 1.44P. A literatura não é clara quanto à origem deste pico. Alguns autores referem-se ao mesmo como referente ao plano $002^{[8-10]}$. Já para outros autores este pico pode ser devido às diferentes conformações das moléculas do sal dentro das galerias da argila $^{[11,2]}$. Para este estudo o interesse está em estudar o pico referente ao plano 001 que está associado ao plano onde existem as moléculas do sal, região aonde acontecem as modificações, havendo intercalação ou esfoliação da argila, pela introdução das moléculas poliméricas.

Como pode ser observado na Figura 4, onde são apresentadas as curvas para a argila pura e os masterbatches, ocorreu intercalação ou esfoliação da argila nos masterbatches. Na Tabela 2 são apresentados os valores dos espaçamentos basais referentes ao plano 001 da argila pura e para os diferentes masterbatches. O MB 0 apresenta um aumento no

Tabela 1. Condições de processamento.

\begin{tabular}{cccc}
\hline Masterbatch & Processamento & Parâmetros da extrusora \\
\cline { 3 - 5 } $\begin{array}{c}\text { Composição } \\
\text { (PE-g-MA/Argila) }\end{array}$ & & Perfil de temperatura $\left({ }^{\circ} \mathbf{C}\right)$ & Velocidade (rpm) \\
\hline MB 0 (65/35) & 1 & $180,190,195,200,230$ & 400 \\
MB 1 (75/25) & 1 & $210,220,220,220,230$ & 500 \\
MB 2 (90/10) & 1 & $210,220,220,220,230$ & 500 \\
& 2 & $180,180,190,200,200$ & 400 \\
\hline
\end{tabular}




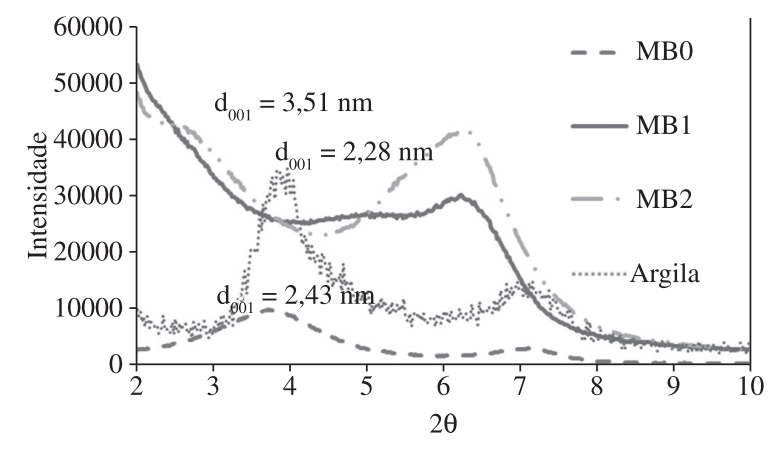

Figura 4. Curvas de difração de raio $X$ para a argila pura e para os masterbatches.

Tabela 2. Valores do espaçamento basal.

\begin{tabular}{ccc}
\hline & $\mathbf{2 \theta}\left({ }^{\circ}\right)$ & $\mathbf{d}_{\mathbf{0 0 1}}(\mathbf{n m})$ \\
\hline Nanomer 1.44P & 3,88 & 2,28 \\
MB 0 (35\%) & 3,64 & 2,43 \\
MB1 $(25 \%)$ & - & - \\
MB2 $(10 \%)$ & $2,52 *$ & 3,51 \\
\hline
\end{tabular}

*Ombro, não é um pico bem definido.

espaçamento basal, sendo associado a uma intercalação das camadas da argila. Para o MB 1 o pico referente ao plano 001 da argila desapareceu e para o MB 2 pode-se ver um pequeno ombro, muito provavelmente devido a uma pequena fração não esfoliada. O aparecimento de ombro na mesma região na difração de raio $\mathrm{X}$ também foi observado por Golebiewski et al. ${ }^{[13]}$. Outros estudos relatam a coexistência de fases esfoliadas e compactadas, passando por vários níveis de delaminação da $\operatorname{argila}^{[14,15]}$. Embora seja esperada uma distribuição de estados de delaminação, os masterbatches com menor teor de argila e processados com maiores temperaturas e maior velocidade de rosca promoveram melhores condições de delaminação das plaquetas da argila. A opção por diluição e reprocessamento promoveu modificações favoráveis de delaminação detectadas pela difração de raio X.

Permeabilidade ao oxigênio: Os resultados da TPO são mostrados na Tabela 3. A presença do compatibilizante utilizado para melhorar a dispersão da argila na blenda influenciou fortemente os resultados de permeabilidade ao oxigênio, possivelmente por afetar a cristalinidade da blenda.
Já os nanocompósitos apresentaram melhoria de barreira ao oxigênio, com valores de TPO significativamente menores, visto que também continham o compatibilizante.

Permeabilidade ao vapor d'água: Estes resultados também estão apresentados na Tabela 3. A mesma influência do compatibilizante foi notada para a TPVA. O PEBDL-g-MA sendo polar, pode aumentar a permeabilidade da blenda ao vapor d'água, embora os valores ainda sejam baixos, pois o polietileno é intrinsecamente uma ótima barreira ao vapor d'água. A presença da argila reduz os valores, mas em menor proporção que para o oxigênio, exatamente pelo fato da matriz já ser uma boa barreira neste caso.

Aplicação do modelo de Nielsen: Os valores relativos do coeficiente de permeabilidade da blenda e dos nanocompósitos foram utilizados para a aplicação do modelo de Nielsen (Equação 8), para determinação da razão de aspecto $\alpha$. $\mathrm{O}$ modelo trata-se de um estudo a partir de propriedades macroscópicas para a interpretação do desempenho dos nanocompósitos. Para isto, primeiramente foi necessário determinar para cada composição a fração volumétrica $\phi$ da fase impermeável da argila, isto é, da fase inorgânica. Foi previamente obtida por TGA que a fase inorgânica da argila corresponde a $67,4 \%$ de sua massa, sendo então também considerada a massa específica da argila não modificada de $2,86 \mathrm{~g} / \mathrm{cm}^{3[16]}$. A partir da fração volumétrica para cada composição e dos valores relativos de permeabilidade foram encontrados os valores de $\alpha$ a partir de TPO e TPVA (valores de $\alpha$ TPO e $\alpha$ TPVA na Tabela 3 ).

$\mathrm{O} \alpha$ corresponde à razão de aspecto da argila. Considerando que uma nanoplaqueta da argila tem espessura aproximada de $1 \mathrm{~nm}^{[17]}$, os valores obtidos correspondem ao comprimento médio das camadas impermeáveis com cerca de $100 \mathrm{~nm}$. O valor de razão de aspecto encontrado está menor que os relatados na literatura. Ploehn e Liu relatam, a partir de observações por microscopia de força atômica, uma distribuição de razão de aspecto com valor médio de 166 e desvio padrão de 86 para uma argila montmorilonita totalmente esfoliada ${ }^{[17]}$.

Observa-se que os maiores valores foram obtidos para as amostras com 5\% de argila, indicando ser esta composição a que melhor esfoliação apresentou. As condições de processo não mostraram uma tendência consistente.

Tabela 3. Valores do coeficiente de permeabilidade e da razão de aspecto $\alpha$.

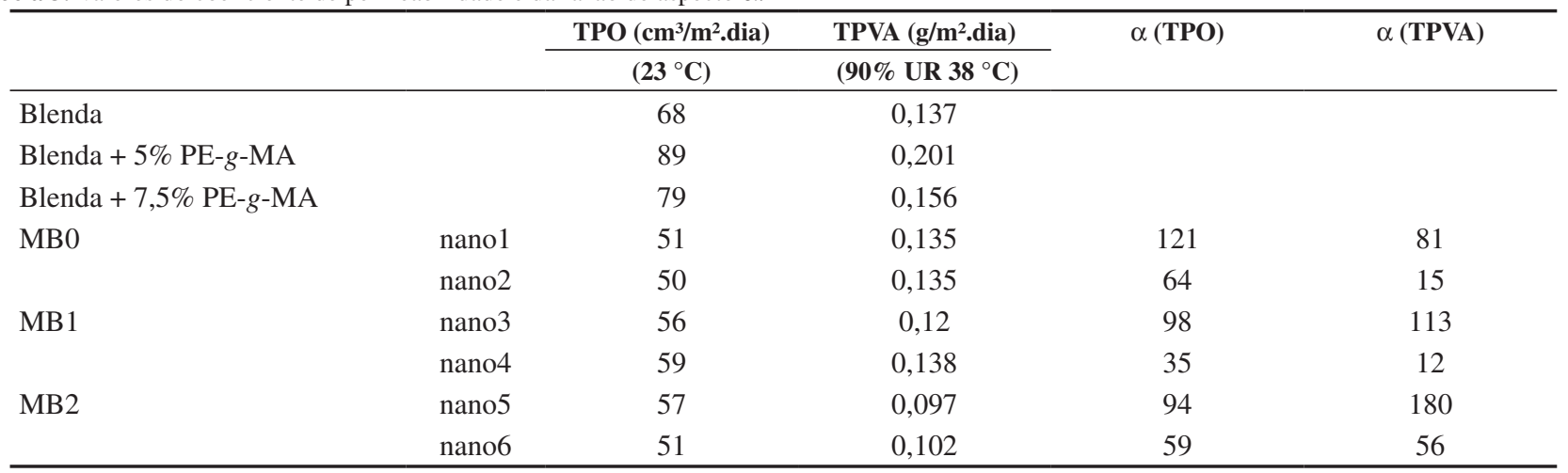


A microscopia eletrônica transmissão indicou para a composição de 5\% melhor delaminação da argila, conforme Figura 5. Observam-se regiões com aglomerados de argila mais pronunciados na condição com $7,5 \%$ de argila. Embora não seja possível detectar o empacotamento das nanoplaquetas, as lamelas parecem ter dimensões inferiores a $100 \mathrm{~nm}$, confirmando o indicado pelo modelo de Nielsen.

Mesmo para as amostras que apresentaram os maiores valores de $\alpha$, estes estão em torno de 100 , o que é pode ser considerado relativamente baixo para se obter boas propriedades de barreira.

Dois efeitos podem estar ocorrendo: primeiramente a delaminação das plaquetas da argila não foi adequada e um baixo grau de esfoliação foi obtido e em segundo lugar, a argila, originalmente pode ter uma razão de aspecto muito baixa para este tipo de aplicação. A razão de aspecto $\alpha$ próxima de 100 indica que a argila utilizada deve ter uma dimensão lateral não suficiente para aumentar o caminho tortuoso para o gás permeante, mesmo assim foi obtida uma redução de $30 \%$ na permeabilidade de oxigênio em relação à blenda original.

O trabalho mostra a necessidade estudos para detalhar a razão de aspectos de argila para este fim. Na literatura, a

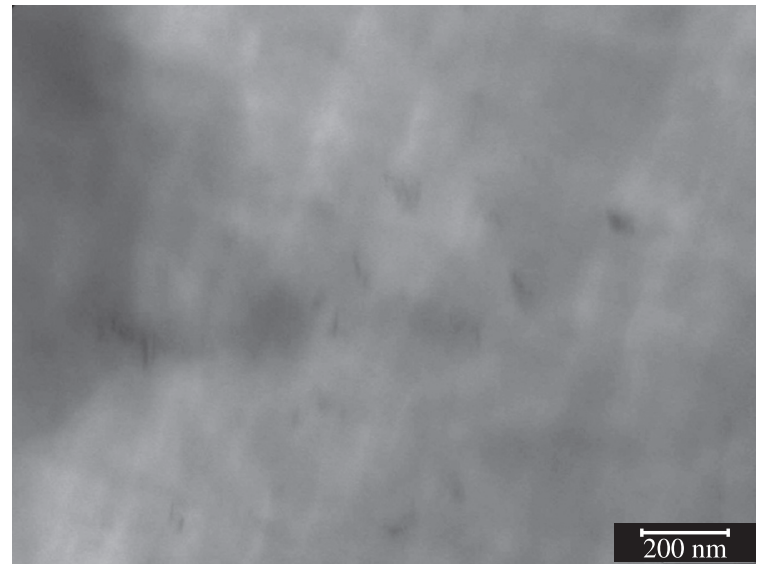

(a)

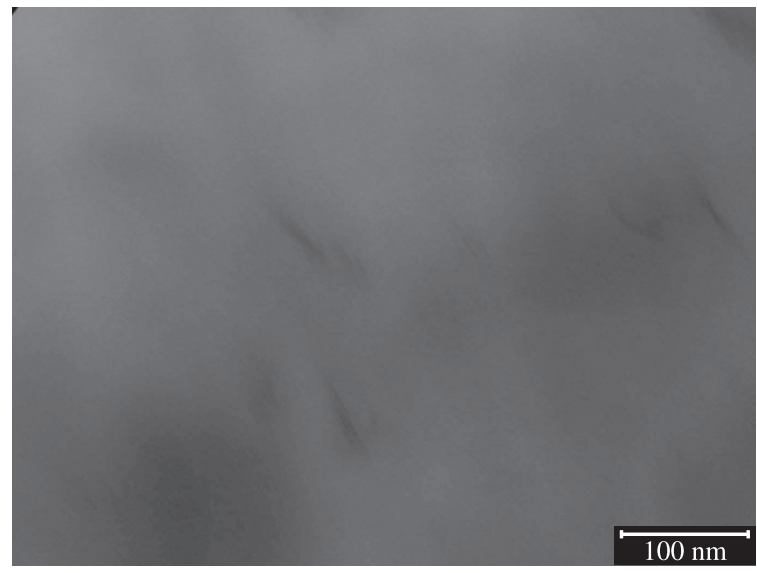

(b) importância da razão de aspecto também foi observada por $\mathrm{Xu}$ e co-autores ${ }^{[18]}$.

Outro aspecto a ser considerado é que o sistema estudado é uma blenda, e que separação de fases entre o PEAD e o PEBDL pode ocorrer. Neste caso os valores de permeabilidade podem ser influenciados, principalmente se a argila provocar alteração na morfologia das fases, ou mesmo apresentar localização preferencial em uma das fases. Pelas micrografias obtidas por microscopia eletrônica de transmissão não foi possível comprovar estas ocorrências.

Propriedades mecânicas e propriedades óticas: As propriedades mecânicas foram pouco alteradas comparandose a blenda original com os nanocompósitos. Na Tabela 4, são apresentados os resultados do módulo de elasticidade e da resistência máxima de tração para os sentidos longitudinal e transversal da extrusão.

A Figura 6 apresenta as curvas de transmissão de luz em espectrofotômetro de UV-Visível. A transparência manteve-se inalterada ou aumentou levemente para os nanocompósitos, sendo um indício de alguma alteração na cristalinidade das blendas poliméricas. A propriedade de transparência é de grande interesse para as aplicações de embalagens flexíveis transparentes.

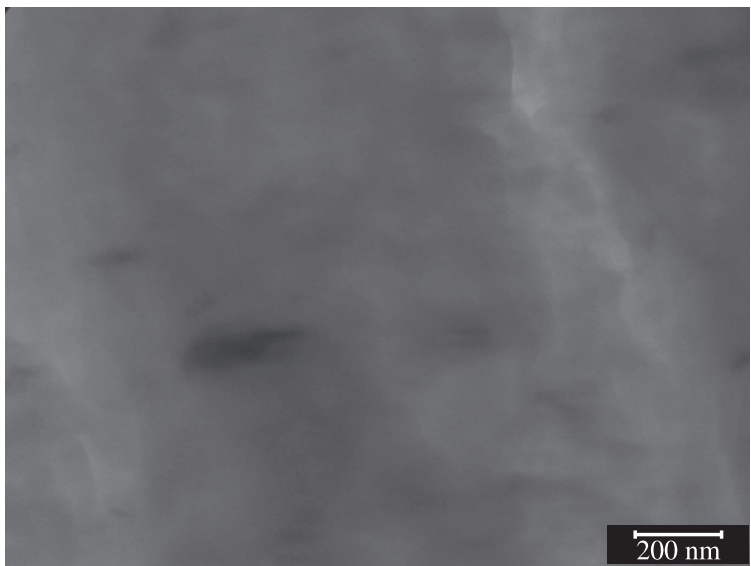

(c)

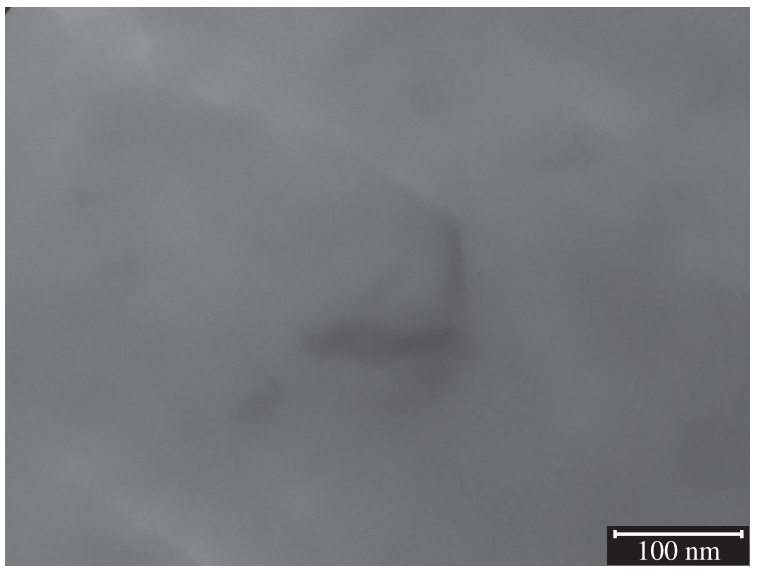

(d)

Figura 5. Fotomicrografia de MET para o nanocompósitos a) e b) com 5\% de argila: nano5; c) e d) com 7,5\% de argila: nano6. 
Tabela 4. Ensaios de tração no sentido longitudinal e transversal de extrusão.

\begin{tabular}{lcccc}
\hline \multicolumn{1}{c}{ Amostra } & $\begin{array}{c}\text { Módulo elástico (MPa) } \\
\text { longitudinal }\end{array}$ & $\begin{array}{c}\text { Tensão máxima (MPa) } \\
\text { longitudinal }\end{array}$ & $\begin{array}{c}\text { Módulo elástico (MPa) } \\
\text { transversal }\end{array}$ & $\begin{array}{c}\text { Tensão máxima (MPa) } \\
\text { transversal }\end{array}$ \\
\hline Blenda & $380 \pm 19$ & $28 \pm 4$ & $334 \pm 25$ & $27 \pm 2$ \\
Blenda + 5\% PE-g-MA & $347 \pm 21$ & $26 \pm 1$ & $480 \pm 17$ & $26 \pm 4$ \\
Blenda + 7,5\% PE-g-MA & $322 \pm 19$ & $25 \pm 2$ & $424 \pm 42$ & $25 \pm 1$ \\
Nano 3 & $255 \pm 25$ & $25 \pm 3$ & $283 \pm 16$ & $27 \pm 2$ \\
Nano 4 & $320 \pm 20$ & $29 \pm 2$ & $333 \pm 25$ & $33 \pm 2$ \\
Nano 5 & $362 \pm 30$ & $27 \pm 3$ & $396 \pm 15$ & $31 \pm 3$ \\
Nano 6 & $336 \pm 24$ & $35 \pm 3$ & $342 \pm 33$ & $29 \pm 2$ \\
\hline
\end{tabular}
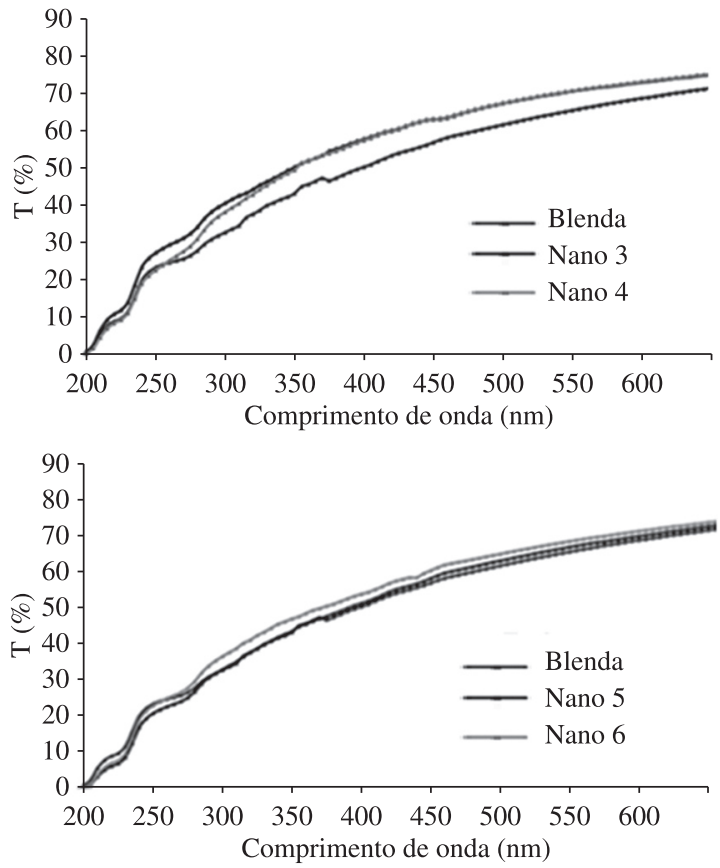

Figura 6. Transmissão de luz em porcentagem (\%T) para a blenda e os nanocompósitos com 5 e 7,5\% de argila.

\section{Conclusão}

A aplicação do modelo de Nielsen demonstrou ser um recurso útil para a avaliação do grau de esfoliação das argilas. Trata-se de um estudo a partir de propriedades macroscópicas para a interpretação do desempenho dos nanocompósitos. Este recurso indicou que o sistema estudado apresenta duas ocorrências importantes: esfoliação insuficiente e baixa razão de aspecto da argila. Portanto, pode-se concluir que aumentos significativos de propriedades de barreira são possíveis pela obtenção de uma boa esfoliação, mas também se faz necessária a utilização de argilas com alta razão de aspecto das nanoplaquetas.

\section{Agradecimentos}

Os autores agradecem à CAPES pelo apoio financeiro, à Cromex pelo fornecimento das matérias primas e preparação dos nanocompósitos, ao DEMA-UFSCar e ao CETEA/ITAL pela colaboração na realização dos ensaios.

\section{Referências Bibliográficas}

1. Lotti, C.; Isaac, C.; Branciforti, M. C.; Alves, R. M. V.; Liberman, S. \& Bretas, R. E. S. - Eur. Polymer J., 44, p.1346 (2008).

2. Pavlidou, S. \& Papaspyrides, C. D. - Progr. Polymer Sci., 33, p.1119 (2008).

3. Rodolfo Jr., A. \& Mei, L. H. I. - Polímeros: Ciênc Tecnol., 19, p.1 (2009).

4. Bharadwaj, R. K. - Macromolecules, 34, p.9189 (2001).

5. Crank, J. \& Park, G. S. - "Diffusion in Polymers", Academic Press, New York (1968).

6. Utracki, L. A. - "Clay-Containing Polymeric Nanocomposites", Rapra Technology, England (2004).

7. Choudalakis, G. \& Gotsis, A. D. - Eur. Polymer J., 45, p.967 (2009).

8. Pack, S.; Mayu-Si, M.; Koo, J.; Sokolov, J. C.; Koga, T.; Kashiwagi, T. \& Rafailovich, M. H. - Polymer Degradation and Stability, 94, (2008).

9. Araújo, E. M.; Barbosa, R.; Rodrigues A. W. B.; Mello T. J. A. \& ITO, E. N. - Mater. Sci. Eng. A, 445, p.141(2007).

10. Xu, W.; Liang, G.; Zhai, H.; Tang, S.; Hang, G. \& Pan, W. Eur. Polymer J., 39, p.1467 (2003).

11. He, H.; Frost, R.L.; Bostrom, T.; Yuan, P.; Duong, L.; Yang, D.; Xi, Y.; Kloprogge, J. T. - Appl. Clay Sci., 31, p.2621 (2006).

12. Zhou, Q.; Frost, R. L.; He, H.; Xi, Y. \& Liu, H. - J. Colloid Interface Sci., 307, p.357 (2007).

13. Golebiewski, J.; Rozanski, A.; Dzwonkowski, J. \& Galeski, A. - Eur. Polymer J., 44, p.270 (2008).

14. Paul, D. R. \& Robeson, L. M. - Polymer, 49, p.3187 (2008).

15. Ray, S. S. \& Okamoto, M. - Progr. Polymer Sci., 28, p. 1539 (2003).

16. Alexandre, B.; Langevin, D.; Médéric, P.; Aubry, T.; Couderc, H.; Nguyen, Q. T.; Saiter, A. \& Marais, S. - J. Membr. Sci., 328, p.186 (2009).

17. Ploehn, H. J. \& Liu, C. - Ind. Eng. Chem. Res., 45, p.7025 (2006).

18. Xu, B.; Zheng, Q.; Song, Y. \& Shangguan, Y. - Polymer, 47, p.2904 (2006).

Enviado: 17/06/09

Reenviado: 02/12/09

Aceito: 04/12/09

DOI: $10.1590 / \mathrm{S} 0104-14282010005000004$ 